\title{
Non-linear-wave-based excitation of waves on a membrane
}

\section{Heikkila, Jesse}

IEEE Computer Society

2019

Heikkila , J , Lampsijärvi , E , Kontiola , A , Salmi , A \& Haggstrom , E 2019 , Non-linear-wave-based excitation of waves on a membrane . in 2019 IEEE International Ultrasonics Symposium (IUS) . IEEE Computer Society , pp. 166-169, IEEE International Ultrasonics Symposium , Glasgow , United Kingdom , 06/10/2019 . https://doi.org/10.1109/ULTSYM.2019.8925954

http://hdl.handle.net/10138/326283

https://doi.org/10.1109/ULTSYM.2019.8925954

acceptedVersion

Downloaded from Helda, University of Helsinki institutional repository.

This is an electronic reprint of the original article.

This reprint may differ from the original in pagination and typographic detail.

Please cite the original version. 


\section{Non-linear-wave-based excitation of waves on a membrane}

\author{
$1^{\text {st }}$ Jesse Heikkilä \\ Electronics lab., Physics dept. \\ Univ. of Helsinki \\ Helsinki, Finland \\ jesse.t.heikkila@helsinki.fi
}

$5^{\text {th }}$ Edward Hæggström

Electronics lab., Physics dept.

Univ. of Helsinki

Helsinki, Finland

edward.haeggstrom@helsinki.fi

\author{
$2^{\text {nd }}$ Eetu Lampsijärvi \\ Electronics lab., Physics dept. \\ Univ. of Helsinki \\ Helsinki, Finland \\ eetu.lampsijarvi@helsinki.fi
}

\author{
$3^{\text {rd }}$ Antti Kontiola \\ Univ. of Helsinki \\ Helsinki, Finland \\ antti.kontiola@photono.fi
}

\author{
$4^{\text {th }}$ Ari Salmi \\ Electronics lab., Physics dept. \\ Univ. of Helsinki \\ Helsinki, Finland \\ ari.salmi@helsinki.fi
}

\begin{abstract}
Non-linear acoustic waves were used to excite propagating waves on a curved biological membrane. A noncontact method was used to measure the displacement of the membrane at the pickup point. The frequency content, time-offlight, and amplitude of the wave travelling across the membrane at the measurement location were studied as a function of incidence angle of the shockwave for different pressures inside a vessel bounded by the membrane. Detecting the peak frequency of the pickup signal provided the most accurate estimate of the pressure inside the vessel.
\end{abstract}

\section{INTRODUCTION}

Acoustic radiation force from air-coupled focused ultrasound has been used to excite propagating waves on a surface of biological tissue [1-2]. We used a non-linear acoustic wave (shockwave) based excitation to generate a propagating wave that travelled across a curved biological membrane. Our study investigates the robustness of the non-contacting method that determines the pressure inside a vessel bounded by the membrane. Biological tissues commonly exhibit nonlinear stress-strain behavior [3-5], which manifests as increased Young's modulus as a function of increased pressure in the vessel. This affects the velocity and frequency content of the wave propagating on the membrane [6]. Peak frequency and time-of-flight of the propagating wave were studied as a function of incidence angle of the exciting shockwave to find an analysis method that provides accurate results and high sensitivity in the studied case.

\section{MEASUREMENTS}

\section{A. Excitation method}

Non-contact excitation of propagating waves on a membrane was launched with a shockwave created with a triggerable three-electrode high-voltage $1.8 \mathrm{kV}$ spark gap. The shockwave was coupled into a waveguide and guided close to the membrane. The shockwave is followed by turbulent air flow and a propagating air vortex ring created at the end of the waveguide. A shockwave reflector was added to the end of the waveguide to change the direction of the shockwave. This allowed us to separate the shockwave and the vortex ring, because only the shockwave is efficiently reflected.

An experiment was done to demonstrate the behavior of shockwave-based signals. The distance from the waveguide to the membrane was increased in $100 \mu \mathrm{m}$ steps while keeping the excitation location constant. A new excitation and measurement were performed at each distance. Schlieren imaging was used to align the waveguide and the shockwave reflector by verifying the propagation direction of impinging the shockwave and the excitation location on the membrane during the measurement.

\section{B. Experimental setup}

A straight dual-field-lens ( $\mathrm{f}=200 \mathrm{~mm}$ ) stroboscopic schlieren arrangement [7] visualized and measured speed of the shockwave, and the direction of the incidence angle of the shockwave onto the membrane. Stroboscopic measurements revealed the speed of the shockwave travelling towards the membrane. The stroboscopic schlieren setup includes an USBcamera (IDS UI-3480CP), a custom-made LED pulser, a green LED and a signal generator (Tektronix AFG 3252). The LED was pulsed multiple times at $400 \mathrm{kHz}$ for each produced shockwave to determine the shockwave speed from the propagation distance between pulses. The spark gap creating the shockwave was triggered with a thyristor driven by a FET connected to an Atmega328p microcontroller. A current transformer triggered the signal generator feeding the LED pulser with a controlled pulse frequency and delay.

The setup for non-contacting measurements of the wave travelling across the membrane consists of a laser Doppler vibrometer (Polytec OFV-5000 with OFV-534 laser unit) and an oscilloscope (LeCroy HDO6104). The LDV measured the membrane displacement when the propagating wave passes the pickup point and the oscilloscope measured the output signal of the LDV. These measured signals were used to determine the 
amplitude, time-of-flight, and frequency content of the excited wave after it had propagated across the membrane.

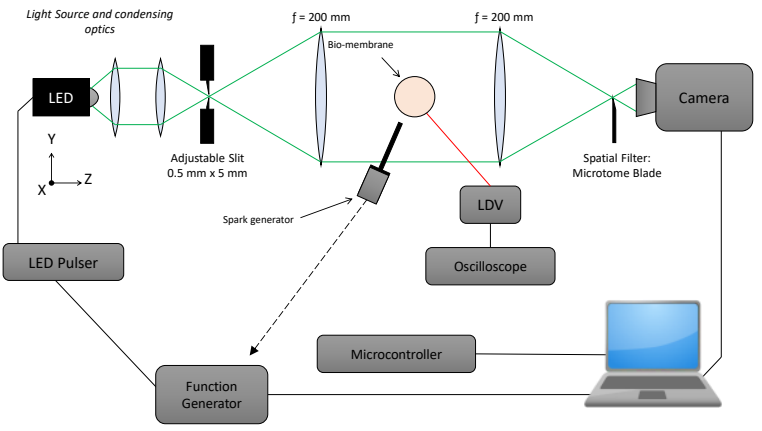

Fig. 1. Illustration of the experimental setup.

The schlieren image in Fig. 2 shows the incident shockwave propagating in air $2 \mu \mathrm{s}$ before contacting with the membrane, whereas Fig. 3 shows the reflected shockwave after the excitation. The incidence angle increases as the shockwave reflector is turned counterclockwise. The incidence angle is $0^{\circ}$ when the propagation of the shockwave is normal to the membrane surface at the desired excitation location.

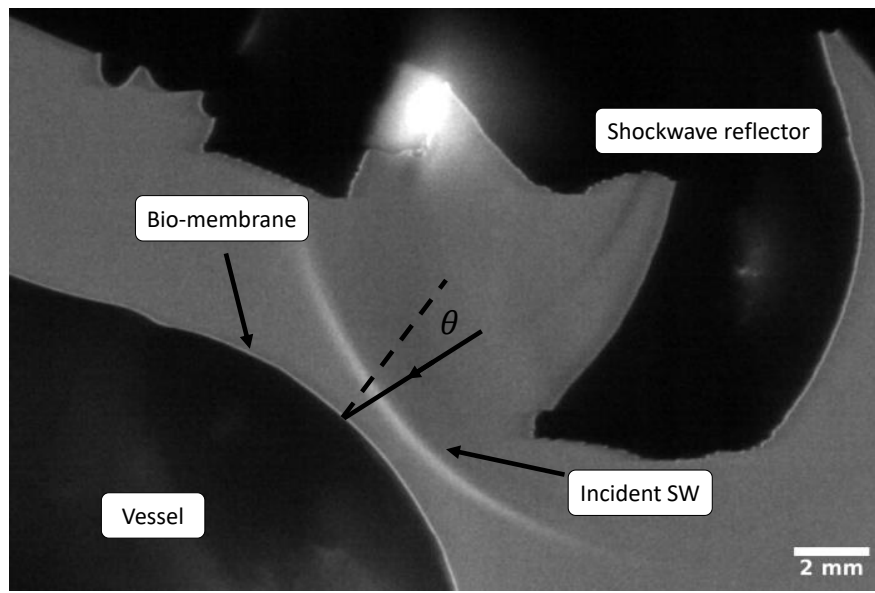

Fig. 2. Schlieren image of the incident shockwave at $\theta=-20^{\circ}$.

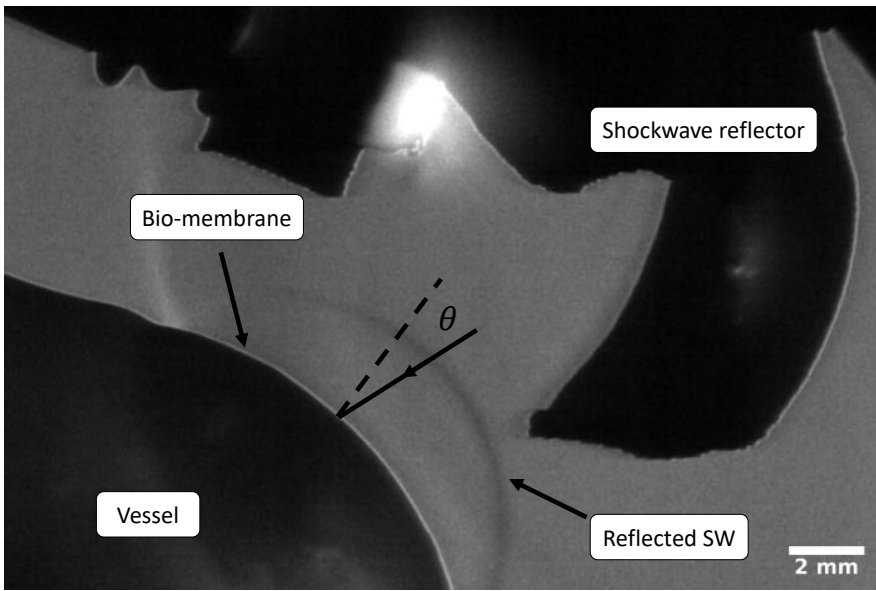

Fig. 3. Schlieren image of the reflected shockwave.
The recorded signals were analyzed in Python 3.6.8. A fast Fourier transform was performed on each signal to detect the peak frequency of the wave travelling across the membrane. The time-of-flight of the signals was calculated by detecting the position of the maximum amplitude in the envelope of the signal. The peak-to-peak displacement amplitude of the membrane at the pickup point was also calculated. These three values were studied separately as a function of incidence angle and pressure inside the vessel. This allowed us to create a calibration curve for estimating the pressure inside the vessel.

\section{RESULTS}

Measurements for shockwave-based excitation as a function of reflector-membrane distance for constant excitation amplitude, location and pressure inside the vessel are shown in Fig. 4. The red line in represents how the signal is expected to move in time for shockwave-based excitation. This increase in time-of-flight of the signal is calculated from the measured speed of the shockwave in Fig. 5. This is possible since only the reflector-membrane distance is altered. Hence the shift in timeof-flight depends only on the speed of the shockwave used as an excitation method.

The maximum increase in propagation time of the shockwave is $20 \mu \mathrm{s}$, which is insignificant compared to the measured time-of-flight of the wave propagating on the membrane. The shockwave-based excitation is robust because aligning the waveguide and the reflector is not critical across repeated measurements as small differences in the propagation distance of the shockwave through air introduces no significant systematic uncertainty in the arrival time of the picked up signal.

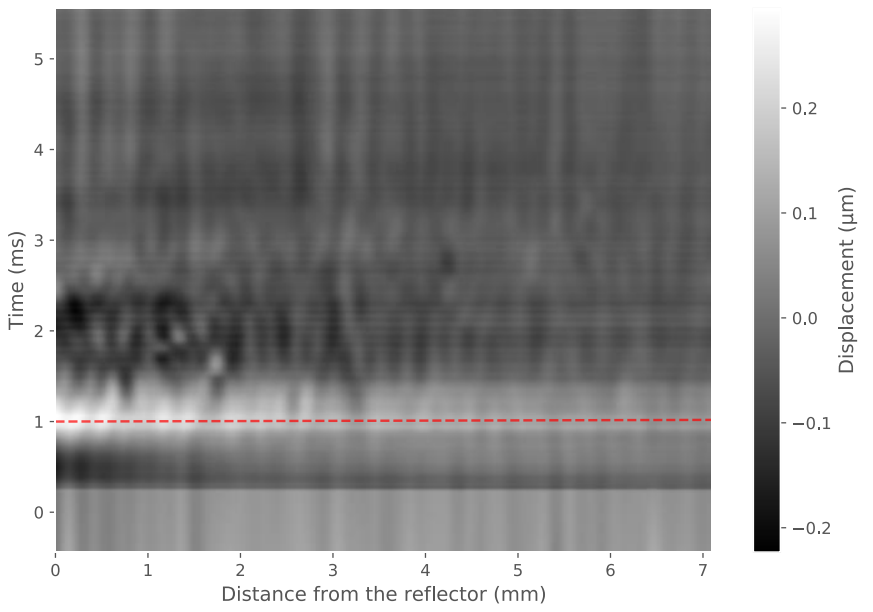

Fig. 4. Locally measured membrane displacement as a function of time-offlight across the membrane and distance between the shockwave reflector and the membrane. 


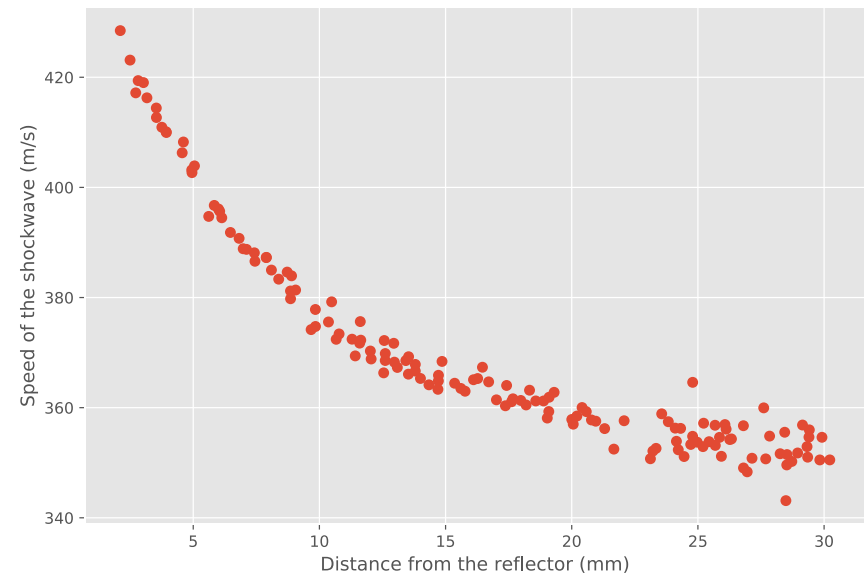

Fig. 5. Shockwave speed in air as a function of propagation distance from the reflector.

Fig. 6 and 7 shows peak frequency and the time-of-flight of the waves on the membrane after propagation distance of $7 \mathrm{~mm}$ across the membrane as a function of incidence angle of the shockwave. The pressure inside the vessel bounded by the biological membrane is presented in each case. Both peak frequency and time-of-flight increase as a function of increasing incidence angle.

The relative change in time-of-flight as a function of incidence angle for each pressure is greater than the relative change in peak frequency. The acceptable uncertainty in angle of incidence is small for the time-of-flight-based analysis. The sensitivity of the measurement decreases with increasing pressure inside the vessel. For example, in the pressure range 20$30 \mathrm{mmHg}$ an uncertainty of $15^{\circ}$ makes it hard to distinguish signals from each other. Altering the angle of incidence does not significantly change the peak frequency. Hence, a peak frequency based analysis method is superior to a time-of-flight method.

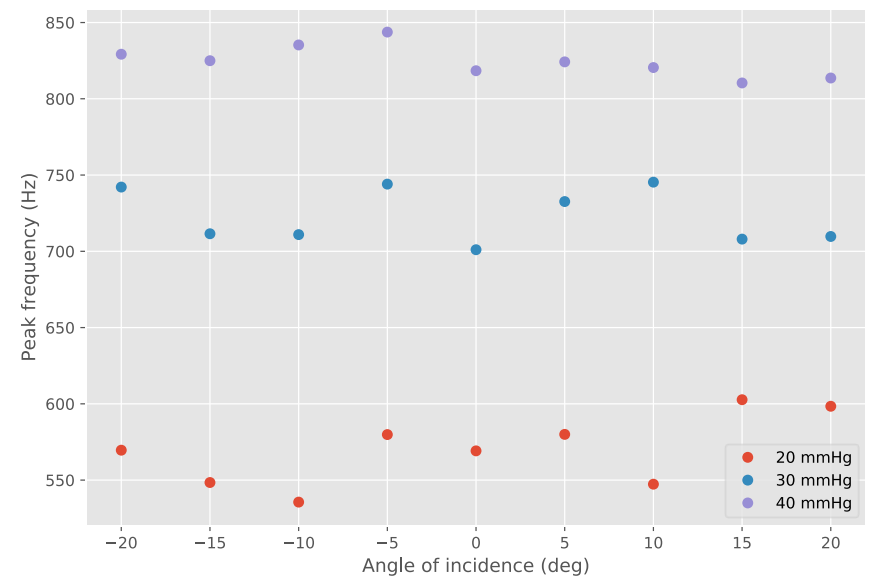

Fig. 6. Peak frequency of the picked up membrane wave as a function of incidence angle.

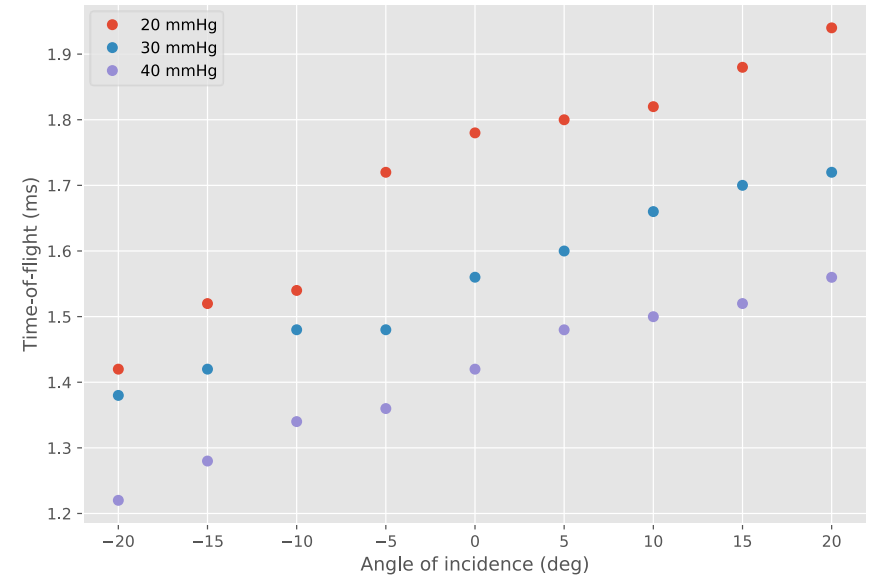

Fig. 7. Time-of-flight of the picked up membrane wave as a function of incidence angle.

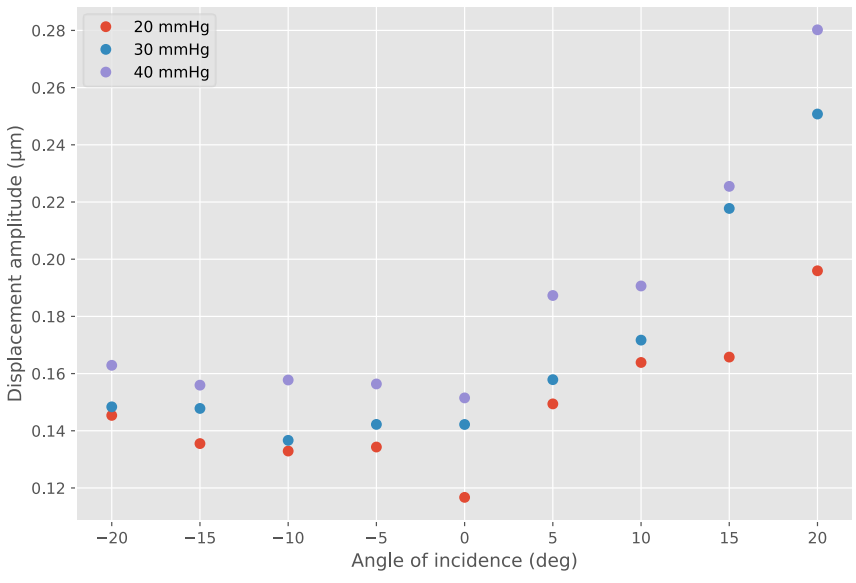

Fig. 8. Peak-to-peak displacement amplitude of the picked up membrane wave as function of incidence angle.

Fig. 8 presents the maximum displacement of the membrane at the pickup point. The relative change in displacement is higher than the relative change in peak frequency and in timeof-flight for half of the studied range of incidence angles. Sensitivity to change in pressure is insignificant with shockwave-based excitation, which renders this method unsuitable for pressure measurements. 


\section{CONCLUSION}

Shockwave-based excitation provided a robust method to excite propagating waves on a curved biological membrane. We used these waves to estimate the pressure inside a vessel bounded by the membrane. The peak frequency of the picked up membrane wave provided the most accurate way to estimate the pressure inside the vessel.

(C) 2021 IEEE. Personal use of this material is permitted. Permission from IEEE must be obtained for all other uses, in any current or future media, including reprinting/republishing this material for advertising or promotional purposes, creating new collective works, for resale or redistribution to servers or lists, or reuse of any copyrighted component of this work in other works.

\section{REFERENCES}

[1] Ambrozinski, L. u., Pelivanov, I., Song, S., Yoon, S. J., Li, D., Gao, L., Shen, T., Wang, R., and O’Donnell, M. (2016a). Air-coupled acoustic radiation force for non-contact generation of broadband mechanical waves in soft media. Applied Physics Letters, 109:043701.

[2] Ambrozinski, L. u., Song, S., Yoon, S. J., Pelivanov, I., Li, D., Gao, L., Shen, T., Wang, R., and O'Donnell, M. (2016b). Acoustic micro-tapping for non-contact $4 \mathrm{~d}$ imaging of tissue elasticity. Scientific Reports, 6:38967.

[3] E Hamilton, K. and Pye, D. (2008). Young's modulus in normal corneas and the effect on applanation tonometry. Optometry and vision science : official publication of the American Academy of Optometry, 85:445-50.

[4] Elsheikh, A., Alhasso, D., and Rama, P. (2008). Biomechanical properties of human and porcine corneas. Experimental eye research, 86:783-90.

[5] Elsheikh, A., Wang, D., and Pye, D. (2007). Determination of the modulus of elasticity of the human cornea. Journal of refractive surgery (Thorofare, N.J. : 1995), 23:808-18.

[6] Viktorov, I. (1970). Rayleigh and Lamb waves: physical theory and applications.

[7] Settles, G. (2001). Schlieren and Shadowgraph Techniques: Visualizing Phenomena in Transparent Media. 